\title{
STRONGLY COTORSION (TORSION-FREE) MODULES AND COTORSION PAIRS
}

\author{
HANGYU YAN
}

\begin{abstract}
In this paper, strongly cotorsion (torsion-free) modules are studied and strongly cotorsion (torsion-free) dimension is introduced. It is shown that every module has a special $\mathcal{S C}_{n}$-preenvelope and an $\mathcal{S} \mathcal{T}_{n^{-}}$cover for any $n \in \mathbb{N}$ based on some results of cotorsion pairs from [9]. Some characterizations of strongly cotorsion (torsion-free) dimension of a module are given.
\end{abstract}

\section{Introduction}

Throughout this paper, all rings are associative with identity and all modules are unitary. For a ring $R$, we write $\operatorname{Mod}-R$ for the category of all right $R$ modules. For a module $M, M^{+}=\operatorname{Hom}_{\mathbb{Z}}(M, \mathbb{Q} / \mathbb{Z})$ will denote the character module of $M$ and $\mathfrak{I}^{-i}(M)\left(\mathfrak{P}^{i}(M)\right)$ will denote the $i$-th cosyzygy (syzygy) of $M$ in $\mathfrak{I}(\mathfrak{P})$, where $\mathfrak{I}(\mathfrak{P})$ is an injective (a projective) resolution of $M$.

We first recall some known notions and facts which we need in the latter sections.

(1) A right $R$-module $M$ is called (Enochs) cotorsion [7] if $\operatorname{Ext}_{R}^{1}(F, M)=0$ for every flat right $R$-module $F$. $M$ is called strongly cotorsion [15] if $\operatorname{Ext}_{R}^{1}(F, M)$ $=0$ for every right $R$-module $F$ of finite flat dimension. A left $R$-module $N$ is called strongly torsion-free [15] if $\operatorname{Tor}_{1}^{R}(F, N)=0$ for every right $R$-module $F$ of finite flat dimension.

(2) Let $M$ be a right $R$-module and $\mathcal{C}$ be a class of right $R$-modules. A homomorphism $\phi: M \rightarrow C$ with $C \in \mathcal{C}$ is called a $\mathcal{C}$-preenvelope of $M[6,8]$ if for any homomorphism $f: M \rightarrow C^{\prime}$ with $C^{\prime} \in \mathcal{C}$, there is a homomorphism $g: C \rightarrow C^{\prime}$ such that $g \phi=f$. Moreover, if the only such $g$ are automorphisms of $C$ when $C=C^{\prime}$ and $f=\phi$, the $\mathcal{C}$-preenvelope $\phi$ is called a $\mathcal{C}$-envelope of $M$. $\mathcal{C}$ is a (pre)enveloping class provided that each module has a $\mathcal{C}$-(pre)envelope. Dually, $\mathcal{C}$-precovers, $\mathcal{C}$-covers, and covering classes of modules can be defined.

Received April 10, 2009; Revised August 13, 2009.

2000 Mathematics Subject Classification. 16E30, 16E10.

Key words and phrases. strongly cotorsion module, strongly torsion-free module, cotorsion pair, strongly cotorsion dimension, strongly torsion-free dimension. 


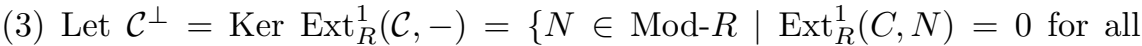
$C \in \mathcal{C}\}$ and ${ }^{\perp} \mathcal{C}=\operatorname{Ker} \operatorname{Ext}_{R}^{1}(-, \mathcal{C})=\left\{N \in \operatorname{Mod}-R \mid \operatorname{Ext}_{R}^{1}(N, C)=0\right.$ for all $C \in \mathcal{C}\}$. A $\mathcal{C}$-preenvelope $\phi: M \rightarrow C$ is called special if $\phi$ is monic and $\operatorname{coker} \phi \in{ }^{\perp} \mathcal{C}$. Dually, the notion of a special $\mathcal{C}$-precover of a module $M$ can be defined.

(4) Let $\mathscr{A}, \mathscr{B} \subseteq$ Mod- $R$. The pair $(\mathscr{A}, \mathscr{B})$ is called a cotorsion pair (or cotorsion theory) $[8,9,11]$ if $\mathscr{A}={ }^{\perp} \mathscr{B}$ and $\mathscr{B}=\mathscr{A}^{\perp}$. Let $\mathscr{C}$ be a class of $R$-modules. Following [9], $\mathfrak{E}_{\mathscr{C}}=\left({ }^{\perp}\left(\mathscr{C}^{\perp}\right), \mathscr{C}^{\perp}\right)$ is called the cotorsion pair generated by $\mathscr{C}$, and $\mathfrak{V}_{\mathscr{C}}=\left({ }^{\perp} \mathscr{C},\left({ }^{\perp} \mathscr{C}\right)^{\perp}\right)$ is called the cotorsion pair cogenerated by $\mathscr{C}$. A cotorsion pair $\mathfrak{E}=(\mathscr{A}, \mathscr{B})$ is called complete if each module has a special $\mathscr{B}$-preenvelope $\left(\mathscr{A}\right.$-precover) and hereditary if $\operatorname{Ext}_{R}^{i}(A, B)=0$ for all $i \geq 1, A \in \mathscr{A}$ and $B \in \mathscr{B}$. $\mathfrak{E}$ is called perfect provided that $\mathscr{A}$ is a covering class and $\mathscr{B}$ is an enveloping class. $\mathfrak{E}$ is called closed provided that the class $\mathscr{A}$ is closed under direct limits in Mod- $R$. Let $\mathcal{S}$ be a set of modules, the cotorsion pair $\left({ }^{\perp}\left(\mathcal{S}^{\perp}\right), \mathcal{S}^{\perp}\right)$ is complete [9].

(5) Let $\mathscr{C}$ be a class of $R$-modules. $\mathscr{C}$ is coresolving provided that $\mathscr{C}$ contains all injective modules, is closed under extensions and $C \in \mathscr{C}$ whenever $0 \rightarrow A \rightarrow$ $B \rightarrow C \rightarrow 0$ is a short exact sequence such that $A, B \in \mathscr{C}$. Dually, $\mathscr{C}$ is resolving provided that $\mathscr{C}$ contains all projective modules, is closed under extensions and $A \in \mathscr{C}$ whenever $0 \rightarrow A \rightarrow B \rightarrow C \rightarrow 0$ is a short exact sequence such that $B, C \in \mathscr{C}$. For a cotorsion pair $\mathfrak{E}=(\mathscr{A}, \mathscr{B})$, it is well known that $\mathfrak{E}$ is hereditary if and only if $\mathscr{A}$ is resolving or $\mathscr{B}$ is coresolving.

Let $\mathscr{C}$ be a class of $R$-modules. For a cardinal $\kappa$, we denote by $\mathscr{C} \leq \kappa$ the subclass of $\mathscr{C}$ consisting of the modules possessing a projective resolution containing only $\leq \kappa$-generated modules. We denote by $\mathcal{F}_{n}\left(\mathcal{I}_{n}, \mathcal{P}_{n}\right)$ the class of all right $R$-modules of flat (injective, projective) dimension $\leq n$, where $n$ is a nonnegative integer. Furthermore, $\mathcal{F}(\mathcal{P})$ will denote the class of all right $R$-modules of finite flat (projective) dimension. We use $f d(M)$ and $i d(M)$ to denote the usual flat and injective dimensions of a module $M$ respectively. $r F P D(R)$ will denote the supremum of the projective dimensions of all right $R$-modules of finite projective dimension.

In Section 2, we introduce the concept of strongly cotorsion dimension. We show that every module has a special $\mathcal{S C}_{n}$-preenvelope for any $n \in \mathbb{N}$. Some characterizations of strongly cotorsion dimension of a module are given.

In Section 3, we introduce the concept of strongly torsion-free dimension. We show that every module has an $\mathcal{S} \mathcal{T} \mathcal{F}_{n}$-cover for any $n \in \mathbb{N}$. Some characterizations of strongly torsion-free dimension of a module are given.

For unexplained concepts, notions and facts, we refer the reader to $[1,3,4$, $5,10]$.

\section{Strongly cotorsion modules}

We start with the following definition. 
Definition 2.1. Given a right $R$-module $M$. Let $\operatorname{scd}(M)=\inf \{n$ : there exists an exact sequence $0 \rightarrow M \rightarrow C_{0} \rightarrow C_{1} \rightarrow \cdots \rightarrow C_{n} \rightarrow 0$ of right $R$-modules, where each $C_{i}$ is strongly cotorsion $\}$ and call $\operatorname{scd}(M)$ the strongly cotorsion dimension of $M$. If no such $n$ exists, set $\operatorname{scd}(M)=\infty$.

In what follows, $\mathcal{S C}_{n}$ denotes the class of all right $R$-modules of strongly cotorsion dimension $\leq n$, where $n$ is a nonnegative integer. Clearly, $\mathcal{S C}_{0}$ is the class of all strongly cotorsion right $R$-modules.

Lemma 2.2. Let $R$ be a ring and $M$ a right $R$-module. Then $M$ is strongly cotorsion if and only if $\operatorname{Ext}_{R}^{i}(F, M)=0$ for every $F \in \mathcal{F}$ and $i \geq 1$.

Proof. The assertion is clear, since all syzygies of modules in $\mathcal{F}$ are again in $\mathcal{F}$.

Proposition 2.3. Let $R$ be a ring. The class $\mathcal{S C}_{0}$ is coresolving.

Proof. By Lemma 2.2, the cotorsion pair $\left({ }^{\perp} \mathcal{S C}_{0}, \mathcal{S C}_{0}\right)$ is hereditary, so the claim follows by [9, Lemma 2.2.10].

Lemma 2.4 ([13, Lemma 1.5(3)]). Let $R$ be a ring and $n \in \mathbb{N}$. Let $\kappa=$ $\operatorname{card}(R)+\aleph_{0}$. Then $\left(\mathcal{F}_{n},\left(\mathcal{F}_{n}\right)^{\perp}\right)$ is a cotorsion pair generated by $\mathcal{F}_{n}^{\leq \kappa}$.

Theorem 2.5. Let $R$ be a ring. Then $\left({ }^{\perp} \mathcal{S C}_{0}, \mathcal{S C}_{0}\right)$ is a hereditary complete cotorsion pair and every right $R$-module has a special $\mathcal{S C}_{0}$-preenvelope. In particular, $\left({ }^{\perp} \mathcal{S C}_{0}, \mathcal{S C}_{0}\right)$ is a cotorsion pair generated by $\bigcup_{n \in \mathbb{N}} \mathcal{F}_{n}^{\leq \kappa}$, where $\kappa=\operatorname{card}(R)+\aleph_{0}$.

Proof. By definition, $\mathcal{S C}_{0}=\mathcal{F}^{\perp}$, thus $\left({ }^{\perp} \mathcal{S C}_{0}, \mathcal{S C}_{0}\right)=\left({ }^{\perp}\left(\mathcal{F}^{\perp}\right), \mathcal{F}^{\perp}\right)$ is a cotorsion pair. Since $\mathcal{S C}_{0}$ is a coresolving class by Proposition 2.3, the cotorsion pair $\left({ }^{\perp} \mathcal{S C}_{0}, \mathcal{S C}_{0}\right)$ is hereditary. By Lemma 2.4, $\mathcal{S C}_{0}=\mathcal{F}^{\perp}=\left(\bigcup_{n \in \mathbb{N}} \mathcal{F}_{n}\right)^{\perp}=$ $\bigcap_{n \in \mathbb{N}} \mathcal{F}_{n}^{\perp}=\bigcap_{n \in \mathbb{N}} \mathcal{F}_{n}^{\leq \kappa}{ }^{\perp}=\left(\bigcup_{n \in \mathbb{N}} \mathcal{F}_{n}^{\leq \kappa}\right)^{\perp}$, where $\kappa=\operatorname{card}(R)+\aleph_{0}$. So $\left({ }^{\perp} \mathcal{S C}_{0}\right.$, $\left.\mathcal{S C}_{0}\right)$ is a cotorsion pair generated by $\bigcup_{n \in \mathbb{N}} \mathcal{F}_{n}^{\leq \kappa}$. It is easy to see that $\bigcup_{n \in \mathbb{N}} \mathcal{F}_{n}^{\leq \kappa}$ has a representative set of elements, then the cotorsion pair $\left({ }^{\perp} \mathcal{S C}_{0}, \mathcal{S C}_{0}\right)$ is complete, and hence every right $R$-module has a special $\mathcal{S C}_{0}$-preenvelope.

We are now ready to compute the strongly cotorsion dimension of a non-zero module. We start with the following definition.

Definition 2.6. Let $M$ be a non-zero right $R$-module, $\delta(M)=\inf \{n \in \mathbb{N} \mid$ $\operatorname{Ext}_{R}^{n+1}(F, M)=0$ for all $\left.F \in \mathcal{F}\right\}$, and $\lambda(M)=\sup \left\{n \in \mathbb{N} \mid \operatorname{Ext}_{R}^{n}(F, M) \neq 0\right.$ for some $F \in \mathcal{F}$ \}.

Proposition 2.7. Let $R$ be a ring and $M$ a non-zero right $R$-module. Then $\operatorname{scd}(M)=\delta(M)=\lambda(M)$.

Proof. The equality $\delta(M)=\lambda(M)$ is obvious. If $\operatorname{scd}(M)=\infty, \delta(M) \leq \operatorname{scd}(M)$ is obvious. Suppose $\operatorname{scd}(M)=n$, i.e., there is an exact sequence $0 \rightarrow M \rightarrow$ $C_{0} \rightarrow \cdots \rightarrow C_{n} \rightarrow 0$ of right $R$-modules with each $C_{i}$ strongly cotorsion. 
For any $F \in \mathcal{F}$, since $\operatorname{Ext}_{R}^{j}\left(F, C_{i}\right)=0$ for any $i$ and $j \geq 1$, by dimension shifting, $\operatorname{Ext}_{R}^{n+1}(F, M) \cong \operatorname{Ext}_{R}^{1}\left(F, C_{n}\right)=0, \delta(M) \leq n=\operatorname{scd}(M)$ in this case. So $\delta(M) \leq \operatorname{scd}(M)$ always holds. To prove $\operatorname{scd}(M) \leq \delta(M)$, it is enough to prove $\operatorname{scd}(M) \leq \delta(M)$ if $\delta(M)$ is finite. Let $\delta(M)=m$, by dimension shifting, $\operatorname{Ext}_{R}^{1}\left(F, \mathfrak{I}^{-m}(\bar{M})\right) \cong \operatorname{Ext}_{R}^{m+1}(F, M)=0$ for every $F \in \mathcal{F}$, thus $\mathfrak{I}^{-m}(M)$ is strongly cotorsion. Hence $M$ has an exact sequence $0 \rightarrow M \rightarrow E_{0} \rightarrow E_{1} \rightarrow$ $\cdots \rightarrow E_{m-1} \rightarrow C_{m} \rightarrow 0$ such that $C_{m}$ is strongly cotorsion and $E_{i}$ is injective for any $i \in\{0,1, \ldots, m-1\}$. Since $\mathcal{I}_{0} \subseteq \mathcal{S C}_{0}, \operatorname{scd}(M) \leq m$ by definition, i.e., $\operatorname{scd}(M) \leq \delta(M)$. So $\operatorname{scd}(M)=\delta(M)$.

Let $M$ be a module and $\mathcal{C}$ be a class of modules. Recall that $M$ is $\mathcal{C}$ filtered, provided that there are an ordinal $\mu$ and a continuous chain of modules, $\left(M_{\alpha} \mid \alpha \leq \mu\right)$, consisting of submodules of $M$ such that $M=M_{\mu}$, and each of the modules $M_{\alpha+1} / M_{\alpha}(\alpha<\mu)$ is isomorphic to an element of $\mathcal{C}$. A continuous chain of modules $\left(M_{\alpha} \mid \alpha \leq \mu\right)$ is a sequence of modules satisfying $M_{0}=0, M_{\alpha} \subseteq$ $M_{\alpha+1}$ for all $\alpha<\mu$ and $M_{\alpha}=\bigcup_{\beta<\alpha} M_{\beta}$ for all limit ordinals $\alpha \leq \mu$ (see [9, Definition 3.1.1]).

For example, if $\mathcal{C}$ is the class of all simple $R$-modules, then the $\mathcal{C}$-filtered $R$-modules coincide with the semiartinian $R$-modules.

The following lemma due to Eklof gives an important sufficient condition for the vanishing of Ext.

Lemma 2.8 (Eklof Lemma). Let $N$ be a module and $M$ be $a^{\perp} N$-filtered module. Then $M \in{ }^{\perp} N$.

Lemma 2.9 ([9, Corollary 3.2.4]). Let $R$ be a ring and $\mathcal{S}$ a set of modules containing $R$. Then the class ${ }^{\perp}\left(\mathcal{S}^{\perp}\right)$ consists of all direct summands of $\mathcal{S}$-filtered modules.

Theorem 2.10. Let $R$ be a ring and $n \in \mathbb{N}$, and $\kappa=\operatorname{card}(R)+\aleph_{0}$. Then the following are equivalent for a non-zero right $R$-module $M$ :

(1) $\operatorname{scd}(M) \leq n$.

(2) $\delta(M) \leq n$.

(3) $\lambda(M) \leq n$.

(4) $\inf \left\{m \mid \operatorname{Ext}_{R}^{m+1}(F, M)=0\right.$ for all $\left.F \in \bigcup_{i \in \mathbb{N}} \mathcal{F}_{i}^{\leq \kappa}\right\} \leq n$.

(5) $\sup \left\{m \mid \operatorname{Ext}_{R}^{m}(F, M) \neq 0\right.$ for some $\left.F \in \bigcup_{i \in \mathbb{N}} \mathcal{F}_{i}^{\leq \kappa}\right\} \leq n$.

(6) $\mathfrak{I}^{-n}(M)$ is strongly cotorsion.

(7) There is an exact sequence $0 \rightarrow M \rightarrow C_{0} \rightarrow \cdots \rightarrow C_{n} \rightarrow 0$ of right $R$-modules with each $C_{i}$ strongly cotorsion.

(8) If $0 \rightarrow M \rightarrow L_{0} \rightarrow \cdots \rightarrow L_{n-1} \rightarrow W \rightarrow 0$ is an exact sequence of right $R$-modules with each $L_{i}$ strongly cotorsion, then $W$ is strongly cotorsion.

Proof. (1) $\Longleftrightarrow(2) \Longleftrightarrow$ (3) follows from Proposition 2.7.

$(1) \Longleftrightarrow(6)$ is easy to see from the proof of Proposition 2.7.

$(1) \Longleftrightarrow(7)$ is obvious by definition. 
$(6) \Longrightarrow(7)$. Let $0 \rightarrow M \rightarrow E_{0} \rightarrow \cdots \rightarrow E_{n-1} \rightarrow \mathfrak{I}^{-n}(M) \rightarrow 0$ be an exact sequence with $E_{0}, E_{1}, \ldots, E_{n-1}$ injective, by hypothesis, the exact sequence $0 \rightarrow M \rightarrow E_{0} \rightarrow \cdots \rightarrow E_{n-1} \rightarrow \mathfrak{I}^{-n}(M) \rightarrow 0$ satisfies the condition of (7).

$(7) \Longrightarrow(8)$. By hypothesis, $\operatorname{scd}(M) \leq n$ and so $\operatorname{Ext}_{R}^{n+1}(F, M)=0$ for every $F \in \mathcal{F}$. Let $0 \rightarrow M \rightarrow L_{0} \rightarrow \cdots \rightarrow L_{n-1} \rightarrow N \rightarrow 0$ be an exact sequence of right $R$-modules with each $L_{i}$ strongly cotorsion. For any $F \in \mathcal{F}$, since $\operatorname{Ext}_{R}^{k}\left(F, L_{i}\right)=0$ for any $k \geq 1$, by dimension shifting, we have $\operatorname{Ext}_{R}^{1}(F, N) \cong$ $\operatorname{Ext}_{R}^{n+1}(F, M)=0$, i.e., $N$ is strongly cotorsion.

$(8) \Longrightarrow(6)$ is obvious because $M$ has an exact sequence $0 \rightarrow M \rightarrow E_{0} \rightarrow$ $\cdots \rightarrow E_{n-1} \rightarrow \mathfrak{I}^{-n}(M) \rightarrow 0$ with $E_{0}, E_{1}, \ldots, E_{n-1}$ injective.

$(2) \Longrightarrow(4)$ is trivial because $\bigcup_{i \in \mathbb{N}} \mathcal{F}_{i}^{\leq \kappa} \subseteq \mathcal{F}$.

$(4) \Longrightarrow(2)$. For any $F \in \mathcal{F}$, there exists $m \in \mathbb{N}$ such that $F \in \mathcal{F}_{m}$. By Lemmas 2.4 and $2.9, F$ is a direct summand of an $\mathcal{F}_{m}^{\leq \kappa}$-filtered module. By hypothesis and Lemma 2.8, $\operatorname{Ext}_{R}^{n+1}(F, M)=0$ and so $\delta(M) \leq n$.

The proof of $(3) \Longleftrightarrow(5)$ is similar to that of $(2) \Longleftrightarrow(4)$.

Proposition 2.11. Let $0 \rightarrow M_{1} \rightarrow M_{2} \rightarrow M_{3} \rightarrow 0$ be an exact sequence of right $R$-modules. Assume $\operatorname{scd}\left(M_{1}\right)=n$. Then, for any integer $m \geq n$, $\operatorname{scd}\left(M_{2}\right) \leq m \Longleftrightarrow \operatorname{scd}\left(M_{3}\right) \leq m$.

Proof. If $M_{1}=0$, we are done. Suppose $M_{1} \neq 0$. Consider a following exact and commutative diagram of right $R$-modules:

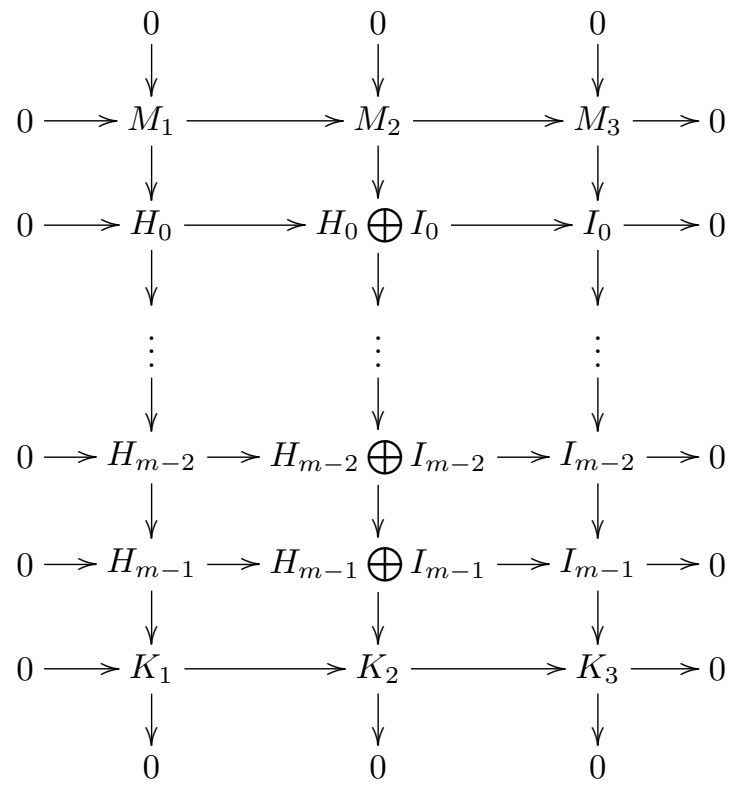

where $H_{i}$ and $I_{i}$ are injective $(0 \leq i \leq m-1)$. It follows from $\operatorname{scd}\left(M_{1}\right)=n$ and Theorem 2.10 that $K_{1}$ is strongly cotorsion. Hence $K_{2}$ is strongly cotorsion 
if and only if $K_{3}$ is strongly cotorsion by Proposition 2.3. This completes the proof.

The following proposition shows that the notion of strongly cotorsion dimension is similar to that of injective dimension.

Proposition 2.12. Let $R$ be a ring and $0 \rightarrow A \rightarrow B \rightarrow C \rightarrow 0$ a short exact sequence of right $R$-modules. Then

(1) $\operatorname{scd}(C) \leq \max \{\operatorname{scd}(A)-1, \operatorname{scd}(B)\}$. If $\operatorname{scd}(B)<\operatorname{scd}(A)$, then $\operatorname{scd}(C)=$ $\operatorname{scd}(A)-1 ;$ if $\operatorname{scd}(B)>\operatorname{scd}(A)$, then $\operatorname{scd}(C)=\operatorname{scd}(B)$.

(2) $\operatorname{scd}(B) \leq \max \{\operatorname{scd}(A), \operatorname{scd}(C)\}$. If $\operatorname{scd}(A) \leq \operatorname{scd}(C)$, then $\operatorname{scd}(B)=$ $\operatorname{scd}(C)$; if $\operatorname{scd}(C) \leq \operatorname{scd}(A)-2$, then $\operatorname{scd}(B)=\operatorname{scd}(A)$. In particular, $\operatorname{scd}(C)=\operatorname{scd}(B)$ if $A$ is strongly cotorsion.

(3) $\operatorname{scd}(A) \leq \max \{\operatorname{scd}(C)+1, \operatorname{scd}(B)\}$. If $\operatorname{scd}(B)<\operatorname{scd}(C)$, then $\operatorname{scd}(A)=$ $\operatorname{scd}(C)+1 ;$ if $\operatorname{scd}(B)>\operatorname{scd}(C)$, then $\operatorname{scd}(A)=\operatorname{scd}(B)$.

Proof. For any $F \in \mathcal{F}$, we have the long exact sequence

$$
\begin{aligned}
\operatorname{Ext}_{R}^{1}(F, A) & \rightarrow \operatorname{Ext}_{R}^{1}(F, B) \rightarrow \operatorname{Ext}_{R}^{1}(F, C) \rightarrow \operatorname{Ext}_{R}^{2}(F, A) \\
& \rightarrow \cdots \rightarrow \operatorname{Ext}_{R}^{i}(F, C) \rightarrow \operatorname{Ext}_{R}^{i+1}(F, A) \rightarrow \cdots .
\end{aligned}
$$

If one of $A, B$ and $C$ is zero, we are done. If each of $A, B$ and $C$ is not zero, it is easy to get $(1),(2),(3)$ by Proposition 2.7 and the above long exact sequence.

By Proposition 2.12, we immediately have the following corollary.

Corollary 2.13. Let $R$ be a ring and $0 \rightarrow A \rightarrow B \rightarrow C \rightarrow 0$ a short exact sequence of right $R$-modules. If two of $\operatorname{scd}(A), \operatorname{scd}(B)$ and $\operatorname{scd}(C)$ are finite, then so is the third.

Obviously, $\mathcal{F} \subseteq{ }^{\perp} \mathcal{S C}_{0}$. The following proposition gives a criterion when the equality holds.

Proposition 2.14. Let $R$ be a ring. Then $\mathcal{F}={ }^{\perp} \mathcal{S C}_{0}$ if and only if $\mathcal{F}$ is closed under direct sums.

Proof. " $\Longrightarrow$ " is trivial because ${ }^{\perp} \mathcal{S C}_{0}$ is closed under direct sums.

" $\Longleftarrow . "$ Since $\mathcal{F}$ is closed under direct sums, there exists $n \in \mathbb{N}$ such that $\mathcal{F}=\mathcal{F}_{n}$. Thus $\left({ }^{\perp} \mathcal{S C}_{0}, \mathcal{S C}_{0}\right)=\left({ }^{\perp} \mathcal{S C}_{0}, \mathcal{F}^{\perp}\right)=\left({ }^{\perp} \mathcal{S C}_{0}, \mathcal{F}_{n}^{\perp}\right)$, i.e., $\left({ }^{\perp} \mathcal{S C}_{0}, \mathcal{F}_{n}^{\perp}\right)$ is a cotorsion pair. Since $\left(\mathcal{F}_{n}, \mathcal{F}_{n}^{\perp}\right)$ is a cotorsion pair by Lemma $2.4,{ }^{\perp} \mathcal{S C}_{0}=$ $\perp\left(\mathcal{F}_{n}^{\perp}\right)=\mathcal{F}_{n}=\mathcal{F}$.

Proposition 2.15. Let $R$ be a ring and $\left\{M_{i}\right\}_{i \in I}$ a family of right $R$-modules. Then $\operatorname{scd}\left(\prod_{i \in I} M_{i}\right)=\sup \left\{\operatorname{scd}\left(M_{i}\right)\right\}_{i \in I}$.

Proof. Without loss of generality, we may assume that each $M_{i}$ is non-zero. Since $\operatorname{Ext}_{R}^{j}\left(F, \prod_{i \in I} M_{i}\right) \cong \prod_{i \in I} \operatorname{Ext}_{R}^{j}\left(F, M_{i}\right)$ for any $F \in \mathcal{F}$ and $j \geq 0$, $\sup \left\{\operatorname{scd}\left(M_{i}\right)\right\}_{i \in I} \leq \operatorname{scd}\left(\prod_{i \in I} M_{i}\right)$ by Proposition 2.7. If $\sup \left\{\operatorname{scd}\left(M_{i}\right)\right\}_{i \in I}$ is infinite, we are done. Suppose that $\sup \left\{\operatorname{scd}\left(M_{i}\right)\right\}_{i \in I}$ is finite, let $\sup \left\{\operatorname{scd}\left(M_{i}\right)\right\}_{i \in I}$ 
$=n$, then $\operatorname{scd}\left(M_{i}\right) \leq n$ for every $i \in I$, and so $\operatorname{Ext}_{R}^{n+1}\left(F, M_{i}\right)=0$ for every $F \in \mathcal{F}$ and $i \in I$. Thus $\operatorname{Ext}_{R}^{n+1}\left(F, \prod_{i \in I} M_{i}\right)=0$, i.e., $\operatorname{scd}\left(\prod_{i \in I} M_{i}\right) \leq n$ by Theorem 2.10. Hence $\operatorname{scd}\left(\prod_{i \in I} M_{i}\right) \leq \sup \left\{\operatorname{scd}\left(M_{i}\right)\right\}_{i \in I}$ and so $\operatorname{scd}\left(\prod_{i \in I} M_{i}\right)=$ $\sup \left\{\operatorname{scd}\left(M_{i}\right)\right\}_{i \in I}$ in this case. So the assertion holds.

Proposition 2.16. Let $R$ be a ring and $\kappa=\operatorname{card}(R)+\aleph_{0}$. Then every right strongly cotorsion $R$-module is injective if and only if every right $R$-module is an $\bigcup_{n \in \mathbb{N}} \mathcal{F}_{n}^{\leq \kappa}$-filtered module.

Proof. " $\Longrightarrow$ ". By hypothesis, we have ${ }^{\perp} \mathcal{S C}_{0}={ }^{\perp} \mathcal{I}_{0}=$ Mod- $R$. Since ${ }^{\perp} \mathcal{S C}_{0}=$ ${ }^{\perp}\left(\left(\bigcup_{n \in \mathbb{N}} \mathcal{F}_{n}^{\leq \kappa}\right)^{\perp}\right)$ by Theorem 2.5 , for any $H \in{ }^{\perp} \mathcal{S C}_{0}, H$ is a direct summand of an $\bigcup_{n \in \mathbb{N}} \mathcal{F}_{n}^{\leq \kappa}$-filtered module by Lemma 2.9. So every right $R$-module is a direct summand of an $\bigcup_{n \in \mathbb{N}} \mathcal{F}_{n}^{\leq \kappa}$-filtered module. Then every right $R$-module is an $\bigcup_{n \in \mathbb{N}} \mathcal{F}_{n}^{\leq \kappa}$-filtered module by [9, Lemma 4.2.10] (applied for $\kappa^{+}$).

" $\Longleftarrow$. By hypothesis and the proof of " $\Longrightarrow ",{ }^{\perp} \mathcal{S C}_{0}=$ Mod- $R$ by Lemma 2.9. Since $\left({ }^{\perp} \mathcal{S C}_{0}, \mathcal{S C}_{0}\right)$ is a cotorsion pair, $\mathcal{S C}_{0}=\left({ }^{\perp} \mathcal{S C}_{0}\right)^{\perp}=(\operatorname{Mod}-R)^{\perp}=\mathcal{I}_{0}$, i.e., every right strongly cotorsion $R$-module is injective.

Now, we define the global strongly cotorsion dimension of a ring $R$.

Definition 2.17. Let $R$ be a ring. Define $\operatorname{rSCD}(R)=\sup \{\operatorname{scd}(M) \mid M \in$ Mod- $R\}$. $r S C D(R)$ is called the right global strongly cotorsion dimension of $R$.

Proposition 2.18. Let $R$ be a ring. Then the following are equivalent:

(1) $r S C D(R)=0$.

(2) Every right $R$-module $M$ is strongly cotorsion.

(3) $r F P D(R)=0$.

Proof. (1) $\Longleftrightarrow$ (2) follows from definition.

$(2) \Longrightarrow(3)$. By hypothesis, ${ }^{\perp} \mathcal{S C}_{0}={ }^{\perp}(\operatorname{Mod}-R)=\mathcal{P}_{0}$. Since $\mathcal{S C}_{0}=\mathcal{F}^{\perp}, \mathcal{F} \subseteq$ ${ }^{\perp} \mathcal{S C}_{0}=\mathcal{P}_{0}$. Obviously $\mathcal{P}_{0} \subseteq \mathcal{F}$, thus $\mathcal{F}=\mathcal{P}_{0}$. Therefore, every flat right $R$ module is projective, i.e., $R$ is right perfect, and so $\mathcal{F}=\mathcal{P}$. Thus $\mathcal{P}=\mathcal{P}_{0}$, i.e., $r F P D(R)=0$.

$(3) \Longrightarrow(2)$. Since $\operatorname{rFP} D(R)=0, R$ is right perfect by [2, Theorem 6.3]. Hence $\mathcal{F}=\mathcal{P}=\mathcal{P}_{0}$. Thus $\mathcal{S C}_{0}=\mathcal{F}^{\perp}=\mathcal{P}_{0}^{\perp}=$ Mod- $R$, i.e., every right $R$ module is strongly cotorsion.

Proposition 2.19. Let $R$ be a ring. Then the following are equivalent:

(1) $r S C D(R) \leq 1$.

(2) $\mathcal{S C}_{0}$ is closed under factor modules.

(3) $\mathfrak{I}^{-1}(M)$ is strongly cotorsion for every right $R$-module $M$.

Proof. (1) $\Longrightarrow(2)$. For any $L \in \mathcal{S C}_{0}$ and $K \leq L$, we have the short exact sequence $0 \rightarrow K \rightarrow L \rightarrow L / K \rightarrow 0$ of right $R$-modules. Since $\operatorname{scd}(K) \leq 1$ by hypothesis and $\operatorname{scd}(L)=0, \operatorname{scd}(L / K)=0$ by Proposition 2.12 , i.e., $L / K$ is strongly cotorsion. So $\mathcal{S C}_{0}$ is closed under factor modules. 
$(2) \Longrightarrow(3)$ is trivial.

$(3) \Longrightarrow(1)$ follows from Theorem 2.10.

By Proposition 2.19, we immediately have the following corollary.

Corollary 2.20. Let $R$ be a ring. If $\mathcal{F} \subseteq \mathcal{P}_{1}$, then $\operatorname{rSCD}(R) \leq 1$.

Theorem 2.21. Let $R$ be a ring and $n \geq 1$. Then $\left({ }^{\perp} \mathcal{S C}_{n}, \mathcal{S C}_{n}\right)$ is a hereditary complete cotorsion pair. In particular, it is generated by $\mathfrak{P}^{n}\left(\bigcup_{n \in \mathbb{N}} \mathcal{F}_{n}^{\leq \kappa}\right)$, where $\kappa=\operatorname{card}(R)+\aleph_{0}$.

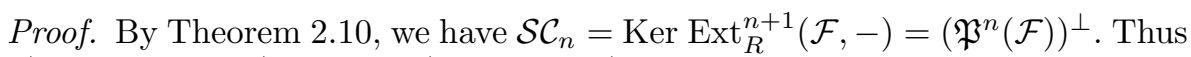
$\left({ }^{\perp} \mathcal{S C}_{n}, \mathcal{S C}_{n}\right)=\left({ }^{\perp}\left(\left(\mathfrak{P}^{n}(\mathcal{F})\right)^{\perp}\right),\left(\mathfrak{P}^{n}(\mathcal{F})\right)^{\perp}\right)$ is a cotorsion pair. By Propositions 2.3 and 2.12 , it is easy to see that $\mathcal{S C}_{n}$ is a coresolving class, so $\left({ }^{\perp} \mathcal{S C}_{n}, \mathcal{S C}_{n}\right)$ is hereditary. Obviously $\mathcal{S C}_{n} \subseteq\left(\mathfrak{P}^{n}\left(\bigcup_{n \in \mathbb{N}} \mathcal{F}_{n}^{\leq \kappa}\right)\right)^{\perp}$ for $\kappa=\operatorname{card}(R)+\aleph_{0}$. For any $C \in\left(\mathfrak{P}^{n}\left(\bigcup_{n \in \mathbb{N}} \mathcal{F}_{n}^{\leq \kappa}\right)\right)^{\perp}$, we have $\operatorname{Ext}_{R}^{1}(M, C)=0$ for every $M \in$ $\mathfrak{P}^{n}\left(\bigcup_{n \in \mathbb{N}} \mathcal{F}_{n}^{\leq \kappa}\right)$, and so $\operatorname{Ext}_{R}^{n+1}(W, C)=0$ for every $W \in \bigcup_{n \in \mathbb{N}} \mathcal{F}_{n}^{\leq \kappa}$. Hence $\operatorname{Ext}_{R}^{1}\left(W, \mathfrak{I}^{-n}(C)\right)=0$ by dimension shifting and so $C \in \mathcal{S C}_{n}$ by Theorem 2.10. Then $\left(\mathfrak{P}^{n}\left(\bigcup_{n \in \mathbb{N}} \mathcal{F}_{n}^{\leq \kappa}\right)\right)^{\perp} \subseteq \mathcal{S C}_{n}$. Therefore, $\mathcal{S C}_{n}=\left(\mathfrak{P}^{n}\left(\bigcup_{n \in \mathbb{N}} \mathcal{F}_{n}^{\leq \kappa}\right)\right)^{\perp}$. Since $\bigcup_{n \in \mathbb{N}} \mathcal{F}_{n}^{\leq \kappa}$ has a representative set of elements, $\mathfrak{P}^{n}\left(\bigcup_{n \in \mathbb{N}} \mathcal{F}_{n}^{\leq \kappa}\right)$ also has a representative set of elements. Thus $\left({ }^{\perp} \mathcal{S C}_{n}, \mathcal{S C}_{n}\right)$ is complete by $[9$, Theorem $3.2 .1]$.

Proposition 2.22. Let $R$ and $S$ be rings. If $C$ is a strongly cotorsion right $S$-module and ${ }_{R} M_{S}$ is a bimodule with $M$ a flat $S$-module. Then $\operatorname{Hom}_{S}(M, C)$ is a strongly cotorsion right $R$-module.

Proof. The proof is modeled on that of [12, Lemma 2.14]. For any right $R$ module $F$ with finite flat dimension, it is easy to see that the flat dimension of $F \otimes_{R} M$ is also finite as a right $S$-module. By hypothesis, we have $\operatorname{Ext}_{S}^{1}\left(F \otimes_{R} M, C\right)=0$. Furthermore, for any projective right $R$-module $P$, we have $\operatorname{Ext}_{S}^{i}(P \otimes M, C)=0$ for any $i>0$ by Lemma 2.2. Then there exists the exact sequence $0 \rightarrow \operatorname{Ext}_{R}^{1}\left(F, \operatorname{Hom}_{S}(M, C)\right) \rightarrow \operatorname{Ext}_{S}^{1}\left(F \otimes_{R} M, C\right)$ by the Grothendieck spectral sequence theorem [14, Theorem 5.8.3]. So

$$
\operatorname{Ext}_{R}^{1}\left(F, \operatorname{Hom}_{S}(M, C)\right)=0
$$

and hence $\operatorname{Hom}_{S}(M, C)$ is a strongly cotorsion right $R$-module.

By Proposition 2.22, we immediately have the following corollaries.

Corollary 2.23. Let $f: R \rightarrow S$ be a ring homomorphism. If $C$ is a strongly cotorsion right $S$-module, then $C$ is a strongly cotorsion right $R$-module.

Corollary 2.24. Let $R$ be a commutative ring and $S$ a multiplicative set of $R$. If $C$ is a strongly cotorsion $S^{-1} R$-module, then $C$ is a strongly cotorsion $R$-module. 
Corollary 2.25. Let $R$ be a commutative ring. If $M$ is a flat $R$-module and $C$ is a strongly cotorsion $R$-module, then $\operatorname{Hom}_{R}(M, C)$ is a strongly cotorsion $R$-module.

Corollary 2.26. If $R$ is a commutative Noetherian ring and $\hat{R}$ is the $I$-adic completion of $R$, where $I$ is a non-trivial ideal of $R$. Assume that $M$ is a strongly cotorsion $\hat{R}$-module, then $M$ is a strongly cotorsion $R$-module.

\section{Strongly torsion-free modules}

We start with the following definition.

Definition 3.1. Given a left $R$-module $N$. Let $\operatorname{st} f d(M)=\inf \{n$ : there exists an exact sequence $0 \rightarrow T_{n} \rightarrow \cdots \rightarrow T_{0} \rightarrow N \rightarrow 0$ of left $R$-modules, where each $T_{i}$ is strongly torsion-free $\}$ and call $\operatorname{st} f d(M)$ the strongly torsion-free dimension of $N$. If no such $n$ exists, set $\operatorname{st} f(N)=\infty$.

In what follows, $\mathcal{S T} \mathcal{F}_{n}$ denotes the class of all left $R$-modules of strongly torsion-free dimension $\leq n$, where $n$ is a nonnegative integer. Clearly, $\mathcal{S} \mathcal{T} \mathcal{F}_{0}$ is the class of all strongly torsion-free left $R$-modules.

The following proposition shows that the notion of strongly cotorsion right $R$-modules can be seen as the dual of strongly torsion-free left $R$-modules in some sense.

Proposition 3.2. Let $R$ be a ring and $N$ a left $R$-module. Then $N$ is strongly torsion-free if and only if $N^{+}$is strongly cotorsion.

Proof. The result follows from the isomorphism $\left(\operatorname{Tor}_{1}^{R}(F, N)\right)^{+} \cong \operatorname{Ext}_{R}^{1}\left(F, N^{+}\right)$ for every $F \in \mathcal{F}$.

Lemma 3.3. Let $R$ be a ring and $N$ a left $R$-module. Then $N$ is strongly torsion-free if and only if $\operatorname{Tor}_{i}^{R}(F, N)=0$ for any $F \in \mathcal{F}$ and $i \geq 1$.

Proof. The proof is similar to that of Lemma 2.2.

Proposition 3.4. Let $R$ be a ring. The class $\mathcal{S} \mathcal{T}_{0}$ is resolving.

Proof. To prove that $\mathcal{S} \mathcal{T} \mathcal{F}_{0}$ is resolving, it suffices to show that $\mathcal{S} \mathcal{T} \mathcal{F}_{0}$ is closed under kernels of epimorphisms because $\mathcal{S} \mathcal{T} \mathcal{F}_{0}=\operatorname{Ker} \operatorname{Tor}_{1}^{R}(\mathcal{F},-)$ by definition. Let $0 \rightarrow A \rightarrow B \rightarrow C \rightarrow 0$ be a short exact sequence of left $R$-modules with $B$ and $C$ strongly torsion-free. For any $F \in \mathcal{F}$, we have the exact sequence $0=\operatorname{Tor}_{2}^{R}(F, C) \rightarrow \operatorname{Tor}_{1}^{R}(F, A) \rightarrow \operatorname{Tor}_{1}^{R}(F, B)=0$ by Lemma 3.3, then $\operatorname{Tor}_{1}^{R}(F, A)=0$ and hence $A \in \mathcal{S} \mathcal{T} \mathcal{F}_{0}$. Thus $\mathcal{S} \mathcal{T} \mathcal{F}_{0}$ is resolving.

Proposition 3.5. Let $R$ be a ring. The class $\mathcal{S} \mathcal{T} \mathcal{F}_{0}$ is closed under direct limits, pure-epimorphic images and pure submodules.

Proof. Since the functor $\operatorname{Tor}_{1}^{R}(F,-)$ commutes with direct limits, $\mathcal{S} \mathcal{T} \mathcal{F}_{0}$ is closed under direct limits. Let $B$ be a strongly torsion-free left $R$-module and $C$ a pure-epimorphic image of $B$, we have the pure exact sequence $0 \rightarrow A \rightarrow$ 
$B \rightarrow C \rightarrow 0$. For any $F \in \mathcal{F}$, we have the exact sequences $0=\operatorname{Tor}_{1}^{R}(F, B) \rightarrow$ $\operatorname{Tor}_{1}^{R}(F, C) \rightarrow F \otimes A \rightarrow F \otimes B \rightarrow F \otimes C \rightarrow 0$ and $0 \rightarrow F \otimes A \rightarrow F \otimes B \rightarrow$ $F \otimes C \rightarrow 0$. Thus $\operatorname{Tor}_{1}^{R}(F, C)=0$ and so $C$ is strongly torsion-free, i.e., $\mathcal{S T} \mathcal{F}_{0}$ is closed under pure-epimorphic images. By Proposition $3.4, \mathcal{S T} \mathcal{F}_{0}$ is closed under pure submodules.

Lemma 3.6 ([9, Theorem 3.2.9]). Let $(\mathcal{A}, \mathcal{B})$ be the cotorsion pair cogenerated by a subclass of pure injective modules. Then $(\mathcal{A}, \mathcal{B})$ is perfect and closed.

Theorem 3.7. Let $R$ be a ring. Then $\left(\mathcal{S} \mathcal{T} \mathcal{F}_{0},\left(\mathcal{S} \mathcal{T} \mathcal{F}_{0}\right)^{\perp}\right)$ is a closed hereditary perfect cotorsion pair, hence $\mathcal{S} \mathcal{T} \mathcal{F}_{0}$ is a covering class.

Proof. For any $F \in \mathcal{F}$, we have $\left(\operatorname{Tor}_{1}^{R}(F, N)\right)^{+} \cong \operatorname{Ext}_{R}^{1}\left(N, F^{+}\right)$. So $N$ is strongly torsion-free if and only if $N \in \perp^{+}\left(\mathcal{F}^{+}\right)$. Thus $\mathcal{S} \mathcal{T} \mathcal{F}_{0}=\perp\left(\mathcal{F}^{+}\right)$, and so $\left(\mathcal{S} \mathcal{T} \mathcal{F}_{0},\left(\mathcal{S} \mathcal{T} \mathcal{F}_{0}\right)^{\perp}\right)=\left({ }^{\perp}\left(\mathcal{F}^{+}\right),\left({ }^{\perp}\left(\mathcal{F}^{+}\right)\right)^{\perp}\right)$ is a cotorsion pair cogenerated by $\mathcal{F}^{+}$. Since $\mathcal{S} \mathcal{T} \mathcal{F}_{0}$ is resolving, $\left(\mathcal{S} \mathcal{T} \mathcal{F}_{0},\left(\mathcal{S} \mathcal{T} \mathcal{F}_{0}\right)^{\perp}\right)$ is hereditary. Since $\mathcal{F}^{+}$is a subclass of pure injective modules, $\left(\mathcal{S} \mathcal{T} \mathcal{F}_{0},\left(\mathcal{S} \mathcal{T} \mathcal{F}_{0}\right)^{\perp}\right)$ is closed and perfect by Lemma 3.6.

Now, we are ready to compute the strongly torsion-free dimension of a nonzero module. We start with the following definition.

Definition 3.8. Let $N$ be a non-zero left $R$-module,

$$
\begin{gathered}
\zeta(N)=\inf \left\{n \in \mathbb{N} \mid \operatorname{Tor}_{n+1}^{R}(F, N)=0 \text { for all } F \in \mathcal{F}\right\}, \text { and } \\
\nu(N)=\sup \left\{n \in \mathbb{N} \mid \operatorname{Tor}_{n}^{R}(F, N) \neq 0 \text { for some } F \in \mathcal{F}\right\} .
\end{gathered}
$$

Proposition 3.9. Let $R$ be a ring and $N$ a non-zero left $R$-module. Then $\operatorname{stfd}(N)=\zeta(N)=\nu(N)$.

Proof. The proof is similar to that of Proposition 2.7.

Theorem 3.10. Let $R$ be a ring and $n \in \mathbb{N}$. Then the following are equivalent for a non-zero left $R$-module $N$ :

(1) $\operatorname{stfd}(N) \leq n$.

(2) $\zeta(N) \leq n$.

(3) $\nu(N) \leq n$.

(4) $\mathfrak{P}^{n}(N)$ is strongly torsion-free.

(5) There is an exact sequence $0 \rightarrow T_{n} \rightarrow \cdots \rightarrow T_{0} \rightarrow N \rightarrow 0$ of left $R$-modules with each $T_{i}$ strongly torsion-free.

(6) If $0 \rightarrow K \rightarrow H_{n-1} \rightarrow \cdots \rightarrow H_{0} \rightarrow N \rightarrow 0$ is an exact sequence of left $R$-modules with each $H_{i}$ strongly torsion-free, then $K$ is strongly torsion-free.

Proof. The proof is similar to that of Theorem 2.10.

The following proposition shows that the notion of strongly torsion-free dimension is similar to that of flat dimension. 
Proposition 3.11. Let $R$ be a ring and $0 \rightarrow A \rightarrow B \rightarrow C \rightarrow 0$ a short exact sequence of left $R$-modules. Then

(1) $\operatorname{stfd}(C) \leq \max \{\operatorname{stfd}(A)+1, \operatorname{stfd}(B)\}$. If stfd( $B)<\operatorname{stfd}(A)$, then stfd $(C)=\operatorname{stfd}(A)+1 ;$ if stfd( $B)>\operatorname{stfd}(A)$, then stfd $(C)=\operatorname{std}(B)$.

(2) $\operatorname{stfd}(B) \leq \max \{\operatorname{stfd}(A)$, stfd( $(C)\}$. If stfd $(C) \leq \operatorname{stfd}(A)$, then st $f d(B)$ $=\operatorname{std}(A)$; if $\operatorname{stfd}(A) \leq \operatorname{stfd}(C)-2$, then $\operatorname{stfd}(B)=\operatorname{stfd}(C)$. In particular, stfd $(B)=\operatorname{stfd}(A)$ if $C$ is strongly torsion-free.

(3) $\operatorname{stfd}(A) \leq \max \{\operatorname{stfd}(C)-1, \operatorname{stfd}(B)\}$. If $\operatorname{stfd}(B)<\operatorname{stfd}(C)$, then $\operatorname{stfd}(A)=\operatorname{stfd}(C)-1$; if $\operatorname{stfd}(B)>\operatorname{stfd}(C)$, then stfd $(A)=\operatorname{stfd}(B)$.

Proof. For any $F \in \mathcal{F}$, we have the long exact sequence $\cdots \rightarrow \operatorname{Tor}_{i+1}^{R}(F, C) \rightarrow$ $\operatorname{Tor}_{i}^{R}(F, A) \rightarrow \cdots \rightarrow \operatorname{Tor}_{2}^{R}(F, C) \rightarrow \operatorname{Tor}_{1}^{R}(F, A) \rightarrow \operatorname{Tor}_{1}^{R}(F, B) \rightarrow \operatorname{Tor}_{1}^{R}(F, C)$. If one of $A, B$ and $C$ is zero, we are done. If each of $A, B$ and $C$ is not zero, it is easy to get (1), (2), (3) by Proposition 3.9 and the above long exact sequence.

By Proposition 3.11, we immediately have the following corollary.

Corollary 3.12. Let $R$ be a ring and $0 \rightarrow A \rightarrow B \rightarrow C \rightarrow 0$ a short exact sequence of left $R$-modules. If two of st $f d(A)$, st $f d(B)$ and st $f d(C)$ are finite, then so is the third.

Proposition 3.13. Let $R$ be a ring and $\left\{N_{i}\right\}_{i \in I}$ a family of left $R$-modules. Then stfd $\left(\bigoplus_{i \in I} N_{i}\right)=\sup \left\{\operatorname{stfd}\left(N_{i}\right)\right\}_{i \in I}$.

Proof. Without loss of generality, we may assume that each $N_{i}$ is non-zero. Since $\operatorname{Tor}_{j}^{R}\left(F, \bigoplus_{i \in I} N_{i}\right) \cong \bigoplus_{i \in I} \operatorname{Tor}_{j}^{R}\left(F, N_{i}\right)$ for any $F \in \mathcal{F}$ and $j \geq 0$, $\sup \left\{\operatorname{stfd}\left(N_{i}\right)\right\}_{i \in I} \leq \operatorname{stfd}\left(\bigoplus_{i \in I} M_{i}\right)$ by Proposition 3.9. If $\sup \left\{\operatorname{stfd}\left(N_{i}\right)\right\}_{i \in I}$ is infinite, we are done. Suppose that $\sup \left\{\operatorname{stf} d\left(N_{i}\right)\right\}_{i \in I}$ is finite. Let

$$
\sup \left\{\operatorname{stfd}\left(N_{i}\right)\right\}_{i \in I}=n .
$$

Then $\operatorname{stfd}\left(N_{i}\right) \leq n$ for every $i \in I$, and so $\operatorname{Tor}_{n+1}^{R}\left(F, N_{i}\right)=0$ for every $F \in \mathcal{F}$ and $i \in I$. Thus $\operatorname{Tor}_{n+1}^{R}\left(F, \bigoplus_{i \in I} N_{i}\right)=0$, stfd $\left(\bigoplus_{i \in I} N_{i}\right) \leq n$ by Theorem 3.10. So $\operatorname{stfd}\left(\bigoplus_{i \in I} N_{i}\right) \leq \sup \left\{\operatorname{stfd}\left(N_{i}\right)\right\}_{i \in I}$. Hence $\operatorname{stfd}\left(\bigoplus_{i \in I} N_{i}\right)=$ $\sup \left\{\operatorname{stfd}\left(N_{i}\right)\right\}_{i \in I}$ in this case. So the assertion holds.

Lemma 3.14 ([9, Lemma 3.2.10]). Let $R$ be a ring. Then the class of all pure injective modules is cosyzygy closed.

Theorem 3.15. Let $R$ be a ring and $n \geq 1$. Then $\left(\mathcal{S T} \mathcal{F}_{n},\left(\mathcal{S T} \mathcal{F}_{n}\right)^{\perp}\right)$ is a closed hereditary perfect cotorsion pair, hence, $\mathcal{S} \mathcal{T} \mathcal{F}_{n}$ is a covering class.

Proof. By Theorem 3.10, we have $\mathcal{S} \mathcal{T} \mathcal{F}_{n}=\operatorname{KerTor}_{n+1}^{R}(\mathcal{F},-)$. Therefore, $\mathcal{S} \mathcal{T} \mathcal{F}_{n}=\operatorname{KerExt}_{R}^{n+1}\left(-, \mathcal{F}^{+}\right)={ }^{\perp}\left(\mathfrak{I}^{-n}\left(\mathcal{F}^{+}\right)\right)$follows from the isomorphism $\left(\operatorname{Tor}_{n+1}^{R}(\mathcal{F}, N)\right)^{+} \cong \operatorname{Ext}_{R}^{n+1}\left(N, \mathcal{F}^{+}\right)$for every $F \in \mathcal{F}$ and $N \in R$-Mod and dimension shifting. By Lemmas 3.6 and 3.14, $\left(\mathcal{S} \mathcal{T} \mathcal{F}_{n},\left(\mathcal{S} \mathcal{T} \mathcal{F}_{n}\right)^{\perp}\right)=\left({ }^{\perp}\left(\mathfrak{I}^{-n}\left(\mathcal{F}^{+}\right)\right)\right.$, $\left.\left({ }^{\perp}\left(\mathfrak{I}^{-n}\left(\mathcal{F}^{+}\right)\right)\right)^{\perp}\right)$ is a perfect and closed cotorsion pair. By Propositions 3.4 and 
3.11 , it is easy to get that $\mathcal{S} \mathcal{T} \mathcal{F}_{n}$ is resolving, thus $\left(\mathcal{S T} \mathcal{F}_{n},\left(\mathcal{S T} \mathcal{F}_{n}\right)^{\perp}\right)$ is hereditary.

Acknowledgements. The author would like to thank the referee for the helpful comments and suggestions.

\section{References}

[1] F. W. Anderson and K. R. Fuller, Rings and Categories of Modules, Springer-Verlag, New York-Heidelberg, 1974.

[2] H. Bass, Finitistic dimension and a homological generalization of semi-primary rings, Trans. Amer. Math. Soc. 95 (1960), 466-488.

[3] P. C. Eklof, S. Shelah, and J. Trlifaj, On the cogeneration of cotorsion pairs, J. Algebra 277 (2004), no. 2, 572-578.

[4] P. C. Eklof and J. Trlifaj, Covers induced by Ext, J. Algebra 231 (2000), no. 2, 640-651.

[5] _ How to make Ext vanish, Bull. Lond. Math. Soc. 33 (2001), no. 1, 41-51.

[6] E. E. Enochs, Injective and flat covers, envelopes and resolvents, Israel J. Math. 39 (1981), no. 3, 189-209.

[7] Flat covers and flat cotorsion modules, Proc. Amer. Math. Soc. 92 (1984), no. $2,179-184$.

[8] E. E. Enochs and O. M. G. Jenda, Relative Homological Algebra, Walter de Gruyter \& Co., Berlin, 2000.

[9] R. Göbel and J. Trlifaj, Approximations and Endomorphism Algebras of Modules, Walter de Gruyter GmbH \& Co. KG, Berlin, 2006.

[10] J. J. Rotman, An Introduction to Homological Algebra, Academic Press, New York, 1979.

[11] L. Salce, Cotorsion theories for abelian groups, Symposia Mathematica, Vol. XXIII (Conf. Abelian Groups and their Relationship to the Theory of Modules, INDAM, Rome, 1977), pp. 11-32, Academic Press, London-New York, 1979.

[12] R. Sazeedeh, Strongly torsion-free modules and local cohomology over Cohen-Macaulay rings, Comm. Algebra 33 (2005), no. 4, 1127-1135.

[13] J. Trlifaj, Infinite dimensional tilting modules and cotorsion pairs, Handbook of tilting theory, 279-321, London Math. Soc. Lecture Note Ser., 332, Cambridge Univ. Press, Cambridge, 2007.

[14] C. A. Weibel, An Introduction to Homological Algebra, Cambridge, Cambridge University Press, 1994.

[15] J. Xu, Flat Covers of Modules, Lecture Notes in Math. 1634, Springer-Verlag, Berlin, 1996.

Department of Mathematics

NANJING UNIVERSITY

NANJing 210093, P. R. China

E-mail address: hyyan07@126.com 